\title{
Computation of dioptric and magnification matrices in ophthalmic lenses
}

\author{
S. Barbero \\ sergio.barbero@csic.es \\ Instituto de Óptica (CSIC), Serrano 121, Madrid, 28006, Spain
}

The diopter power and magnification matrices characterize the first-order properties of ophthalmic lenses for different gaze directions. Therefore an efficient method to compute them is highly valuable in ophthalmic lens design and optical performance simulations. I present a novel method to numerically compute these matrices in ophthalmic lenses comprising any set of arbitrary surfaces. The method is based on computing one base ray, along the gaze direction, and two rays close to it. These two rays are obtained varying a small parameter that indicates their separation from the base ray. The method was validated comparing the results with a single refractive surface where exact solutions are directly obtained.

[DOI: http://dx.doi.org/10.2971/jeos.2014.14023]

Keywords: First-order optics, dioptric power matrix, magnification matrix, ophthalmic lenses

\section{INTRODUCTION AND PROBLEM STATEMENT}

First-order optical properties in axial symmetric systems are well established by paraxial optical theory. Two of these properties are particularly relevant: the optical power and the magnification, which, in this case, are scalar quantities. However some types of modern ophthalmic lenses such as progressive (PALs) [1] or Alvarez-type lenses [2] are non-axially symmetric. As a consequence the power and magnification are described not by scalars but by matrices.

This work tackles the problem of computing the so-called dioptric power and magnification matrices. When looking at different object points, through eyeglasses, the eye rotates. Each gaze direction is described by a base ray, which emerges from the eye rotation center, and reaches an observation point. Different dioptric and magnification matrices are associated to the base rays corresponding to each gaze direction.

One way to compute the dioptric matrix is to trace localized quadratic wavefronts along the base rays through the optical system (see e.g. [3]). An alternative is to trace special rays in the vicinity of each base ray. An interesting case appears when the optical surface has a plane of symmetry and the base ray is contained in that plane. It follows that the dioptric matrix is diagonal, when setting one of the coordinates to lie in the plane of symmetry (see section 23.8 in [4]). Then, the first-order properties are separated for each orthogonal coordinate, and consequently they can be computed using paraxial ray equations for each coordinate. Only then one can justify the proposal made by Bourdoncle et al. [5] arguing that power and astigmatism can be obtained using paraxial rays contained at the tangential and sagittal planes. An alternative, based on tracing 8 differential rays, has been used by other authors $[6,7]$. For these rays the optical path difference is com- puted, from which a second order point eikonal function is constructed, containing the information of the dioptric matrix. The disadvantage of such method is that implies tracing too many rays.

This article proposes a method where the dioptric matrix for lenses comprising any type of refractive surfaces can be computed, with a high degree of accuracy, tracing only three rays: the base ray and two near-by ones. The amount of error in the computations is negligible as will be shown later on. Additionally, the magnification matrix can be simultaneously computed.

In visual optics, there are different available definitions of the magnification matrix (see e.g. discussion at [8]). However, when designing, or analyzing, ophthalmic lenses a relevant definition is that one giving the change of gaze direction when looking at an object through eyeglasses with respect to looking at the same object without eyeglasses. Indeed this concept was used as early as 1611 by Johannes Kepler [9], though only using scalar quantities. The magnification matrix provides information of the geometric distortion of the image with respect to the object when looking through different areas of the eyeglasses. I note that another possible definition of the magnification matrix refers to the ratio between the direction vector differentials in the neighborhood of the base ray looking at an object with and without eyeglasses. This would provide information of the local geometric distortion of the image, so it can be defined as a local magnification.

Figure 1 supplies a geometrical interpretation on how the magnification matrix operates. Typically the eye rotations are measured with respect to the eye rotation center $(C)$. The optical axis of the system formed by the eye rotation center and 
the eyeglasses is represented by the dashed line joining $C$ and point T. Imagine we are looking, through eyeglasses, at a point A. Figure 1 shows the object plane containing A and perpendicular to the optical axis. Point $\mathrm{T}$ is at the intersection of this plane with the optical axis. The position vector of A with respect to $\mathrm{T}$ is denoted by $\vec{r}$. Vector $\vec{k}$ denotes the ray vector of the line joining the eye rotation center $(C)$ with the eyeglasses (green solid line), which after refraction reaches point A. Later, we look at the same point but with the naked eyes. Obviously, the gaze direction changes, defining a line (red dashed) joining $\mathrm{C}$ with $\mathrm{A}$, being $\mathrm{s}$ the distance between both points. $\vec{p}$ denotes the ray vector for the new line of sight without eyeglasses.

The magnification matrix $(\tilde{N})$, which elements are $N_{i j}$, relates the $\mathrm{x}-\mathrm{y}$ projection of both vectors through $\left(k_{x}, k_{y}\right)=\tilde{N}\left(p_{x}, p_{y}\right)^{T}$. Now the vector $\left(p_{x}, p_{y}\right)$ can be computed [10] applying simple trigonometry with $\left(p_{x}, p_{y}\right)=\frac{\vec{r}}{s}$, being $s$ the distance from $\mathrm{C}$ to $\mathrm{A}$. So the previous equation can be rewritten:

$$
s\left(\begin{array}{l}
k_{x} \\
k_{y}
\end{array}\right)=\left(\begin{array}{ll}
N_{11} & N_{12} \\
N_{21} & N_{22}
\end{array}\right)\left(\begin{array}{l}
r_{x} \\
r_{y}
\end{array}\right)
$$

Each gaze direction defines a base ray that emerges from the eye rotation center and reaches, after going through the eyeglasses, the viewing point. The dioptric matrix describes the first order power properties in the vicinity of each base ray.

\section{COMPUTATION SCHEME}

In general, in any optical system the dioptric matrix [11] relates the ray position and direction vectors at image space. For our particular case, it is convenient to introduce a special coordinate system where the $z$ and $z^{\prime}$ axes are defined along the directions of the base ray at the object and image spaces respectively. In doing so the object and image planes are orthogonal to the base ray in both spaces. As a consequence the ray coordinates are reduced to two. The coordinates of a point in the object and image planes are $(x, y)$ and $\left(x^{\prime}, y^{\prime}\right)$ respectively. Here, the object and image space contains the viewing point and the eye rotation center respectively. Also $n^{\prime}$ is the refractive index of the image space. Similarly $(\xi, \eta)$ and $\left(\xi^{\prime}, \eta^{\prime}\right)$ denote the vector directions at the object and image planes. Starting from the point eikonal equations (see Sec. 23.1 [4] and notation from [10]) it is possible to derive the following matrix equation:

$$
\left(\begin{array}{l}
-n^{\prime} \xi^{\prime} \\
-n^{\prime} \eta^{\prime}
\end{array}\right)=\left(\begin{array}{ll}
R_{11} & R_{12} \\
R_{12} & R_{22}
\end{array}\right)\left(\begin{array}{l}
x^{\prime} \\
y^{\prime}
\end{array}\right)
$$

Due to the fact that the dioptric matrix is symmetric there are only three elements in it. Therefore Eq. 2 defines a set of linear equations with three variables: $R_{11}, R_{12}$ and $R_{22}$. The idea is that using Eq. 2 and tracing two neighbor rays close to the base ray a new set of linear equations that provide explicit expressions for $R_{11}, R_{12}$ and $R_{22}$ is attainable.

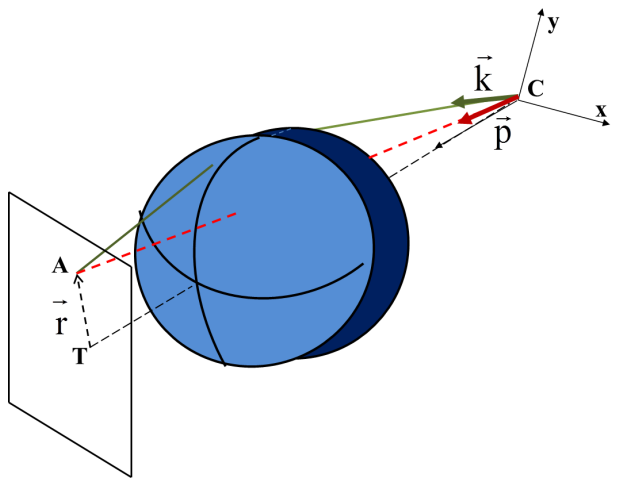

FIG. 1 Magnification matrix scheme.

I proceed as follows:

1. A base ray is traced through the eyeglasses. The intersection point of this ray with the lens surface closer to the object space is denoted by $\left(x_{b}, y_{b}\right)$ an the ray vector by $\left(\xi_{b}, \eta_{b}\right)$.

2. Two points, lying at this surface, in the vicinity of $\left(x_{b}, y_{b}\right)$ are selected with coordinates $\left(x_{1}, y_{1}\right)$ and $\left(x_{2}, y_{2}\right)$ respectively. The coordinates of these rays are: $x_{1}=\delta, y_{1}=0$ and $x_{2}=0, y_{2}=\delta$, being $\delta$ and arbitrarily small quantity.

3. Two rays are constructed at $\left(x_{1}, y_{1}\right)$ and $\left(x_{2}, y_{2}\right)$ both with ray vector $\left(\xi_{b}, \eta_{b}\right)$.

4. After tracing them through the eyeglasses, the corresponding points at the image plane are computed: $\left(x_{1}^{\prime}\right.$, $\left.y_{1}^{\prime}\right)$ and $\left(x_{2}^{\prime}, y_{2}^{\prime}\right)$, in addition with the ray vectors at the image space: $\left(\xi_{1}^{\prime}, \eta_{1}^{\prime}\right)$ and $\left(\xi_{2}^{\prime}, \eta_{2}^{\prime}\right)$.

After computing all these quantities, using Eq. 2 and applying some algebraic calculations the following explicit equation for the variables $R_{11}, R_{12}$ and $R_{22}$ is obtained:

$$
\left(\begin{array}{l}
R_{11} \\
R_{12} \\
R_{12} \\
R_{22}
\end{array}\right)=\left(\begin{array}{cccc}
\frac{y_{2}^{\prime}}{v} & 0 & \frac{-y_{1}^{\prime}}{v} & 0 \\
\frac{-x_{2}^{\prime}}{v} & 0 & \frac{x_{1}^{\prime}}{v} & 0 \\
0 & \frac{y_{2}^{\prime}}{v} & 0 & \frac{-y_{1}^{\prime}}{v} \\
0 & \frac{-x_{2}^{\prime}}{v} & 0 & \frac{x_{1}^{\prime}}{v}
\end{array}\right)\left(\begin{array}{c}
n^{\prime} \xi_{1}^{\prime} \\
n^{\prime} \eta_{1}^{\prime} \\
n^{\prime} \xi_{2}^{\prime} \\
n^{\prime} \eta_{2}^{\prime}
\end{array}\right)
$$

where $v=x_{1}^{\prime} y_{2}^{\prime}-x_{2}^{\prime} y_{1}^{\prime}$. There are two equations providing $R_{12}$. Theoretically both equations should give the same result, but numerical procedures could lead to slightly different results, so it is convenient to take the average of the two quantities obtained from both equations.

Now, these two rays can be also used to compute the magnification matrix. To obtain its four elements at least four equations are needed. The two neighbor rays traced to compute the dioptric matrix are arbitrarily close to the base ray. Therefore the magnification matrix associated to these rays is as close as desired to that of the base ray. Taking this into account, a ma- 
trix equation can be built following the same procedure that was used to obtain Eq. 3:

$$
\left(\begin{array}{c}
N_{11} \\
N_{12} \\
N_{21} \\
N_{22}
\end{array}\right)=\left(\begin{array}{cccc}
\frac{r_{y}^{2}}{u} & 0 & \frac{-r_{y}^{1}}{u} & 0 \\
\frac{-r_{x}^{2}}{u} & 0 & \frac{r_{x}^{1}}{u} & 0 \\
0 & \frac{r_{y}^{2}}{u} & 0 & \frac{-r_{y}^{1}}{u} \\
0 & \frac{-r_{x}^{2}}{u} & 0 & \frac{r_{x}^{1}}{u}
\end{array}\right)\left(\begin{array}{c}
s_{1} k_{x}^{1} \\
s_{1} k_{y}^{1} \\
s_{2} k_{x}^{2} \\
s_{2} k_{y}^{2}
\end{array}\right)
$$

where $u=r_{x}^{1} r_{y}^{2}-r_{x}^{2} r_{y}^{1} .\left(k_{x}^{1}, k_{y}^{1}\right)$ and $\left(k_{x}^{2}, k_{y}^{2}\right)$ are the $\mathrm{x}-\mathrm{y}$ projection of the ray vectors in an absolute coordinate system with center located at point $C$. In the same coordinate system $\left(r_{x}^{1}, r_{y}^{1}\right)$ and $\left(r_{x}^{2}, r_{y}^{2}\right)$ are obtained computing the intersections of these rays with the object plane represented at Figure 1 which contains T and A. $s_{1}$ and $s_{2}$ denote the distances along the line of sight without eyeglasses from the object plane to the optical axis.

I note that although the two rays used to construct Eqs. 3 and 4 are the same, the coordinates of the rays are referred to two different coordinate systems: a relative one depending on the gaze direction for Eq. 3 and an absolute one for Eq. 4 .

To evaluate Eqs. 3 and 4 the two neighbor rays must be carefully selected. It is highly convenient that the two rays lie in planes that are orthogonal one with respect to the other. On one hand the two rays must differ from the base ray only a small amount, but on the other hand if the difference is too small, the matrix values of Eqs. 3 and 4 could lead to numerical singularities that eventually would corrupt the computations. I used the parameter $\delta$ to characterize the separation between the neighbor rays with respect to the base ones. $\delta$ is a distance magnitude $(\mathrm{mm})$ because it gives the separation at the lens surface between the three rays. I empirically evaluated the optimum value of $\delta$.

\section{SPECIAL CASES}

Eqs. 3 and 4 are ill-posed if $v=0$ and $u=0$. Fortunately, when this occurs a geometrical interpretation enable the straightforward computation of first-order properties. For $v=0$ there are three possible cases.

A) $x_{1}^{\prime}=0, y_{1}^{\prime}=0$ AND $x_{2}^{\prime}=0, y_{2}^{\prime}=0$. This implies stigmatic imaging, within a pencil of rays, between the object and the image point. Therefore paraxial optics provides the scalar power and magnification.

B) $x_{1}^{\prime}=0, y_{1}^{\prime}=0$ OR $x_{2}^{\prime}=0, y_{2}^{\prime}=0$. This means that the selected image plane is coincident with one of the focal planes. Therefore, the situation is equivalent to that mentioned at the beginning of the article: i.e. the first-order properties are separated for each orthogonal coordinate, and consequently can be computed using paraxial ray equations for each coordinate.

C) $y_{1} /{ }^{\prime} x_{1}^{\prime}=y_{2}^{\prime} / x_{2}^{\prime}$. This means that the three points configuring the infinitesimal pencil of rays (base ray and two neighbor rays) are imaged onto a line forming $90^{\circ}$ with respect to

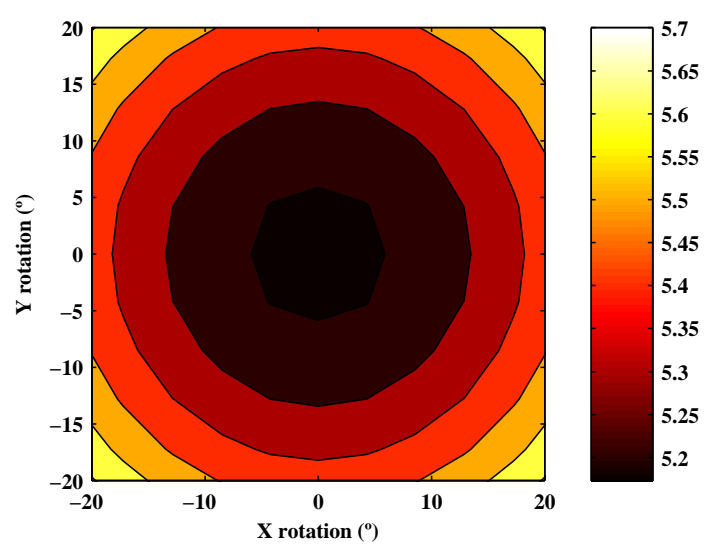

(a)

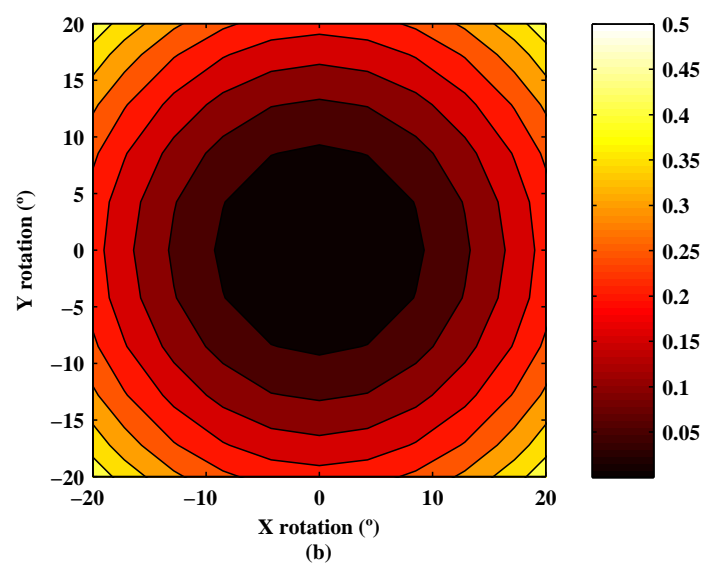

FIG. 2 Nominal (a) mean power (D) and (b) astigmatism (D) as a function of eye rotations.

the base ray at the image space. This case is certainly singular but not impossible. Indeed, even a single spherical mirror can create an image line forming $90^{\circ}$ with respect to the base ray, by selecting an off-axis location of the object point [12]. However, for that case the astigmatism can be obtained from the distance between point $\left(x_{1}^{\prime}, y_{1}^{\prime}\right)$ and $\left(x_{2}^{\prime}, y_{2}^{\prime}\right)$ and the power between the average coordinate of both points and that of the base ray.

\section{SIMULATIONS}

To evaluate the accuracy of the two rays method in computing the dioptric power and magnification matrices I used a canonical test. The test is a single spherical refracting surface. Therefore the dioptric matrix for different gaze directions is diagonal; being the diagonal entries the tangential and sagittal powers, which can be exactly computed using Coddington equations. Figure 2 shows the mean power and astigmatism for different gaze directions as computed with them.

The refractive index of the medium located between the refracting surface and the eye rotation center was 1.5 , and that of the object space was 1 . The distance between the eye rotation center and the refracting surface was $27 \mathrm{~mm}$ (typical value in ophthalmic lens design). The object and image planes were located at $40 \mathrm{~mm}$ and $50 \mathrm{~mm}$ from the refracting sphere. The radius of curvature of the refracting sphere was $80 \mathrm{~mm}$. 


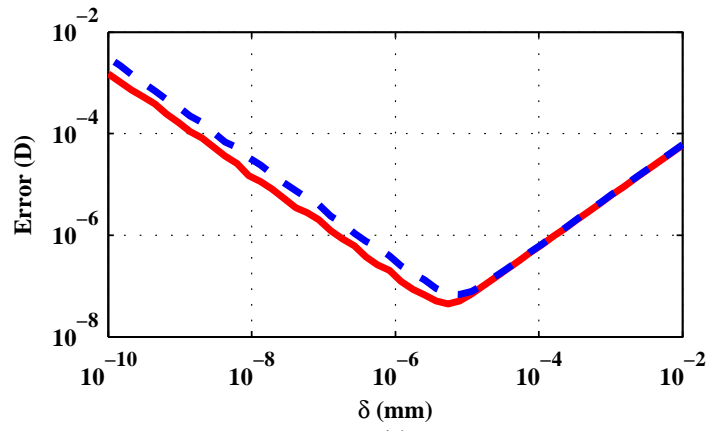

(a)

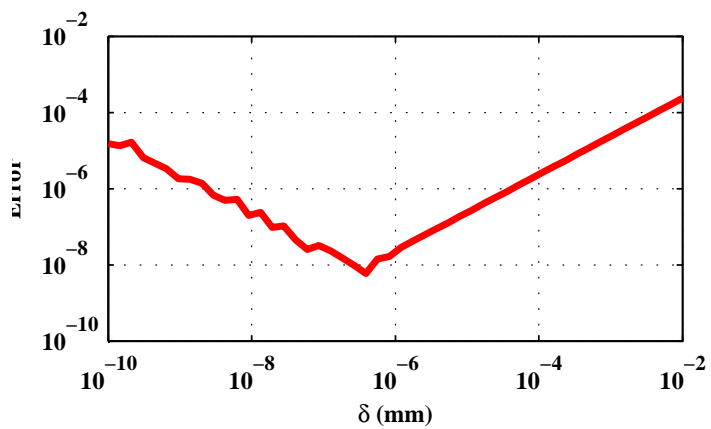

(b)

FIG. 3 (a) Mean power (red solid line) and astigmatism (blue dashed line) errors (D) and (b) root-mean-square deviation in the ray vectors as function of $\delta(\mathrm{mm})$.

The accuracy on the computation of the magnification matrix was evaluated indirectly. Once the magnification matrix was computed, the ray vector (for different gaze directions) with eyeglasses was estimated from the ray vector in the absence of eyeglasses using Eq. 1 and consequently compared to the ray vectors as obtained by exact ray tracing through the spherical refractive surface.

I also explored the optimum separation of the neighbor rays from the base rays with the $\delta$ parameter. The errors were evaluated using the mean absolute error (for different gaze directions) in the mean power (the average of the two principal powers) and in the astigmatism (difference between two principal powers) for different eye rotations. The root-meansquare deviation in the ray vectors was used as an indirect measure of the accuracy in the estimation of the magnification matrix. The fact that the error increases as $\delta$ tends to zero is explained because the problem becomes ill-conditioned as the two neighbor rays provide the same information to that of the base ray.

Figure 3 shows the mean absolute power and astigmatism error (a) and the root-mean-square deviation of the ray vectors (b) as a function of $\delta$. A log-log scale was used.

The graph shows that there exists an optimum value for $\delta$ that is located around $7 \times 10^{-6} \mathrm{~mm}$ for the power and astigmatism errors, while for the ray vectors the minimum error is around $0.3 \times 10^{-6} \mathrm{~mm}$.

Figure 4 shows the error distribution for the mean power and astigmatism for the optimum $\delta=7 \times 10^{-6} \mathrm{~mm}$. The error is completely insignificant for the purpose of ophthalmic lens design
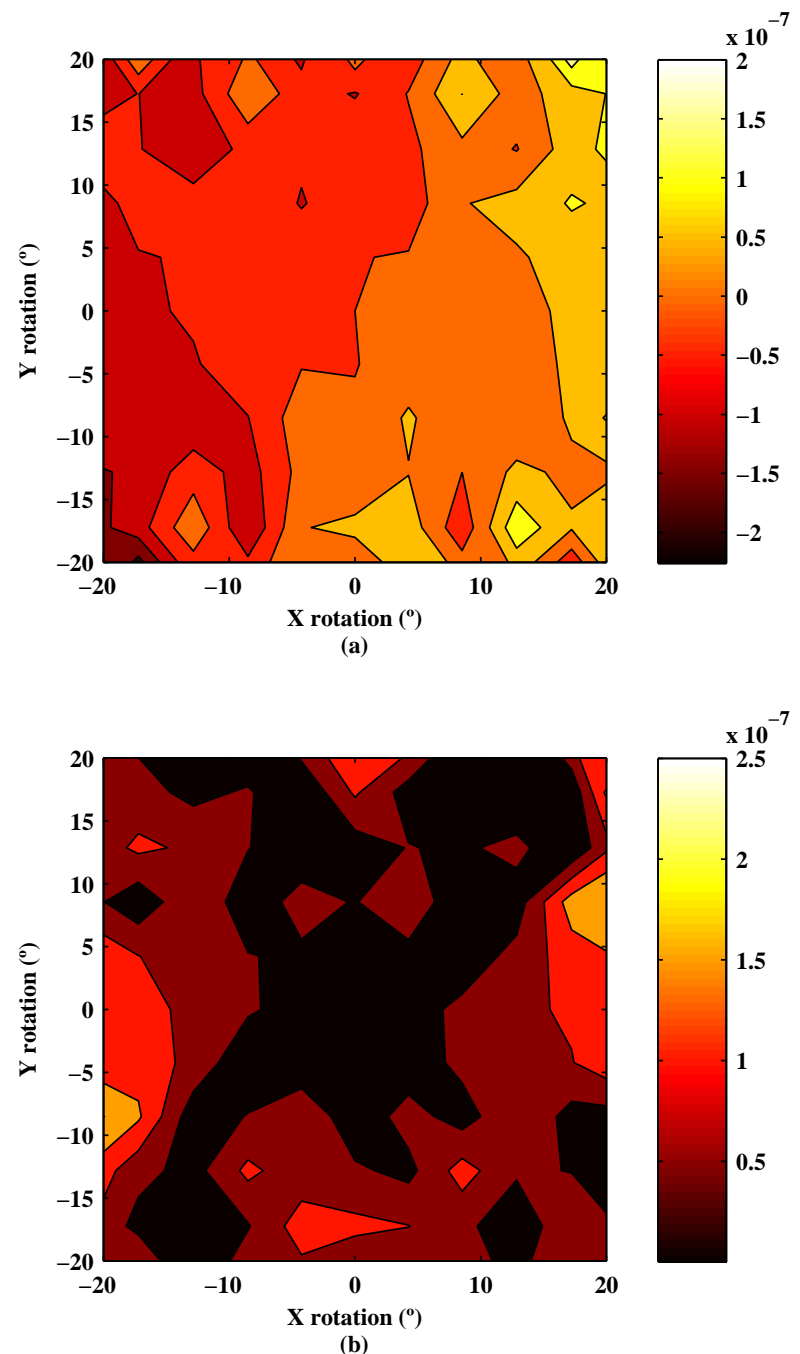

FIG. 4 Computation errors (D) in the mean power (a) and astigmatism (b).

considering that the smallest perceptible image blurring by the human eye is around $0.15 \mathrm{D}$ [13].

Finally Figure 5 shows the $x-y$ ray vector components with and without the refractive surface. The direction cosines exactly computed are compared to the values obtained with the two rays method. Direction vector without eyeglasses are depicted with stars. Exact and estimated direction vector with eyeglasses are depicted with circles and crosses, respectively. The root-mean-square deviation is so small $\left(3.1 \times 10^{-8}\right)$ that in practice can be ignored.

\section{CONCLUSIONS}

I have presented a novel method to compute simultaneously the dioptric power and magnification matrices in ophthalmic lenses. The method is based on tracing two neighbor rays along with the base rays associated to each gaze direction. The computation error associated to this method is completely insignificant for the purposes of ophthalmic lens design when the two neighbor rays are carefully selected. 


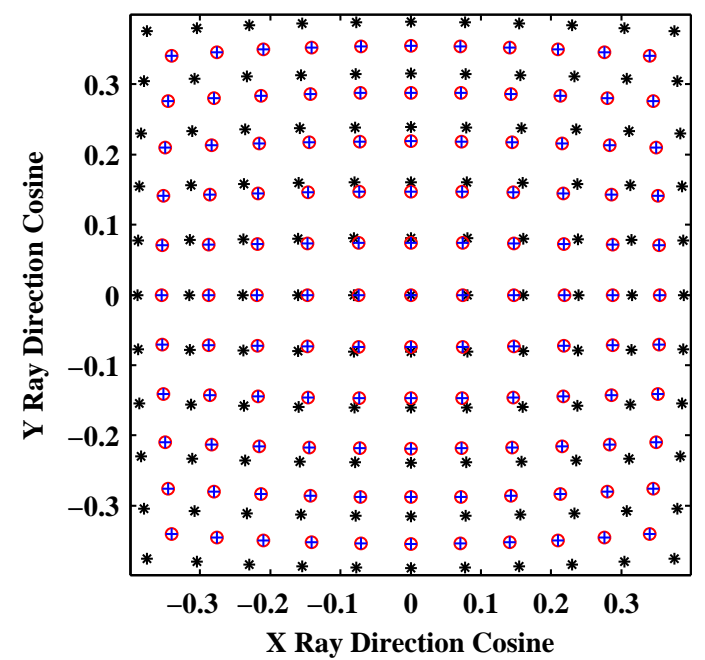

FIG. 5 Ray vectors: (a) without eyeglasses (black stars), (b) exact and estimated with eyeglasses: red circles and blue crosses respectively.

\section{ACKN OWLEDGEMENT}

I thank Jacob Rubinstein and Javier Portilla for their comments and discussion. This work was supported by Spanish Government with Grant: FIS2012-30820.

\section{References}

[1] D. J. Meister, and S. W. Fisher, "Progress in the spectacle correction of presbyopia. Part 1: Design and development of progressive lenses," Clin. Exp. Optom. 91, 240-250 (2008).

[2] S. Barbero, "The Alvarez and Lohmann refractive lenses revisited," Opt. Express. 17, 9376-9390 (2009).
[3] 0. N. Stavroudis, The mathematics of geometrical and physical optics : the k-function and its ramifications (Wiley-VCH, Weinheim, 2006).

[4] A. Walther, The ray and wave theory of lenses (Cambridge University Press, Cambridge, 1995).

[5] B. Bourdoncle, J. P. Chauveau, and J. L. Mercier, "Traps in displaying optical performances of a progressive addition lens," Appl. Opt. 31, 3586-3593 (1992).

[6] S. Barbero, and J. Rubinstein, "Adjustable-focus lenses based on the Alvarez principle," J. Opt. 13, 125705 (2011).

[7] S. Barbero, and J. Rubinstein, "Power-adjustable sphero-cylindrical refractor comprising two lenses," Opt. Eng. 52, 063002-063002 (2013).

[8] W. F. Harris, "Image size magnification and power and dilation factors for optical instruments in general," Ophth. Phy. 0pt. 23, 251-261 (2003).

[9] A. Malet, "Kepler and the telescope," Ann. Sci. 60, 107-136 (2003).

[10] J. Rubinstein, and G. Wolansky, "Differential relations for the imaging coefficients of asymmetric systems," JOSA-A 20, 2365-2369 (2003).

[11] W. F. Harris, "Dioptric power: Its nature and its representation in three- and fourdimensional space," Opt. Vis. Sci. 74, 349-366 (1997).

[12] J. M. Howard, and B. D. Stone, "Imaging a point to a line with a single spherical mirror," Appl. 0pt. 37, 1826-1834 (1998).

[13] R. Legras, N. Chateau, and W. N. Charman, "Assessment of justnoticeable differences for refractive errors and spherical aberration using visual simulation," Opt. Vis. Sci. 81, 718-728 (2004). 